\title{
Especificación y calificación de un procedimiento de soldadura disímil (wps) con proceso gmaw, para láminas de acero de bajo carbono y acero martensítico.
}

\author{
Epecification and Qualification of a Disimil Welding Procedure (wps) with gmaw Process, for \\ Low Carbon and Martensitic Steel Sheets \\ César Mendoza-Gómora ${ }^{a}$, Jesús Reyes-Ortiz ${ }^{b}$,Erick Torres-Cerezo ${ }^{c}$, Carlos Ernesto Borja- \\ Soto $^{d}$, Jorge Zuno-Silva ${ }^{e}$
}

\begin{abstract}
:
This work presents the design of a joint to establish the weldability conditions of two materials used in the automotive industry. The low carbon and martensitic steel have thicknesses of 1 and $1.1 \mathrm{~mm}$ respectively. The design of the joint was carried out considering each of the variables involved in the GMAW process that could affect them. After this process it was found that the sheets of the base material behave much better when the metal transfer system is by short circuit since the heat of contribution is lower. With this design it is possible concluding the WPS that was applied and subsequently qualified complies with all the parameters that allow to obtain a welding with the requested design characteristics.
\end{abstract}

Keywords:

WPS, GMAW, dissimilar weld.

\section{Resumen:}

Este trabajo presenta el diseño de una unión para establecer las condiciones de soldabilidad de dos materiales que se usan en la industria automotriz. El acero bajo carbono y martensítico presentan espesores de 1 y $1.1 \mathrm{~mm}$ respectivamente. El diseño de la soldadura se llevó a cabo considerando cada una de las variables involucradas en el proceso de soldadura GMAW que pudieran afectar. Luego de este proceso se encontró que las láminas del material base se comportan mucho mejor cuando el sistema de transferencia del metal es por corto circuito ya que el calor de aporte es menor. Con este este diseño se puede concluir que la WPS que fue aplicada y posteriormente calificada cumple con todos los parámetros que permiten obtener una unión con las características de diseño solicitadas.

\section{Palabras Clave:}

WPS, GMAW, soldadura disímil.

\footnotetext{
Autor de Correspondencia, Universidad Autónoma del Estado de Hidalgo, Escuela Superior Ciudad Sahagún, Email: cesar_mendoza@uaeh.edu.mx

b Universidad Autónoma del Estado de Hidalgo, Escuela Superior Ciudad Sahagún, Estudiante, Email: jesusreyesortiz@gmail.com

• Universidad Autónoma del Estado de Hidalgo, Escuela Superior Ciudad Sahagún, Estudiante, Email: ericktorrescerezo1@gmail.com

d Universidad Autónoma del Estado de Hidalgo, Escuela Superior Ciudad Sahagún, Email: carlos_borja@uaeh.edu.mx

e Universidad Autónoma del Estado de Hidalgo, Escuela Superior Ciudad Sahagún, Email: jorge_zuno_@uaeh.edu.mx
} 


\section{Introducción}

Los aceros avanzados de alta resistencia como lo es el acero martensítico tiene su aplicación en la industria del transporte debido a su alta resistencia y conformabilidad, esto disminuye el peso de los vehículos que influye directamente en un menor consumo de combustible que a su vez repercute en el mejoramiento del medio ambiente. Otra ventaja del uso de estos materiales es que aumenta el nivel de seguridad del automóvil. No obstante, la unión de estos materiales con aceros de bajo contenido de carbono implica tener un control preciso de las variables operativas del proceso se soldadura. Para ello es indispensable contar con una especificación del procedimiento de soldadura (WPS), es decir, un documento que muestre las variables operativas como lo es la corriente, voltaje, velocidad de desplazamiento, etc., así como el comportamiento mecánico de la unión soldada para garantizar el buen funcionamiento de las estructuras. Ante este panorama, este trabajo presenta un WPS para la unión disímil entre láminas de espesores delgados de un acero bajo carbono con un martensítico, soldadas por el proceso GMAW. 1, 4,5

\section{Materiales y métodos}

Los materiales empleados fueron láminas de acero martensítico (M) y bajo carbono (BC) de 1.1 y $1.0 \mathrm{~mm}$ de espesor respectivamente. El material de aporte fue un electrodo ER70S-6 con un diámetro de $0.9 \mathrm{~mm}$. La composición química de los materiales se muestra en la Tabla 1.

Placas con dimensiones de $200 \mathrm{~mm} \times 100 \mathrm{~mm} \times 1.1 \mathrm{~mm}$, tanto de acero bajo carbono y martensítico fueron maquinadas de acuerdo a los requerimientos de la junta a tope. La Figura 1 muestra el diseño de la junta.

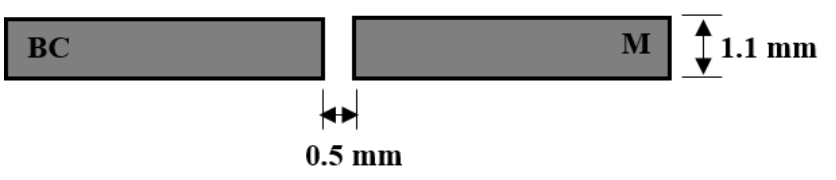

Figura 1. Diseño de junta para la unión disímil.

Se utilizó el proceso de soldadura GMAW, y se usó como gas de protección $98 \% A r-2 \% \mathrm{O}_{2}$. Placas de aluminio fueron utilizadas para disminuir el efecto de la distorsión. La Figura 2 muestra el arreglo experimental y la Tabla 2 muestra los parámetros de soldadura utilizados para la unión.

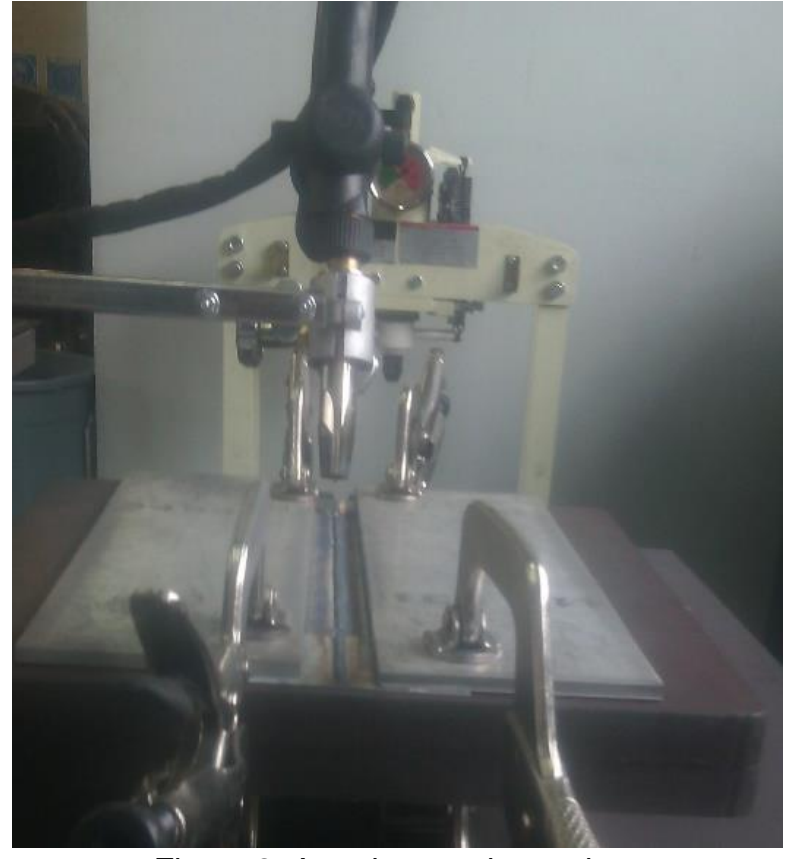

Figura 2. Arreglo experimental.

Tabla 2. Variables operativas utilizadas en las juntas soldadas.

\begin{tabular}{ccccc}
\hline $\begin{array}{c}\text { Corrie } \\
\text { nte (A) }\end{array}$ & $\begin{array}{c}\text { Volta } \\
\text { je } \\
(\mathrm{V})\end{array}$ & $\begin{array}{c}\text { Velocida } \\
\text { d de } \\
\text { alimentac } \\
\text { ión } \\
\left(\mathrm{mm} \mathrm{s}^{-1}\right)\end{array}$ & $\begin{array}{c}\text { Velocidad } \\
\text { de } \\
\text { desplazami } \\
\text { ento } \\
\left(\mathrm{mm} \mathrm{s}^{-1}\right)\end{array}$ & $\begin{array}{c}\text { Distan } \\
\text { cia de } \\
\text { trabajo } \\
(\mathbf{m m})\end{array}$ \\
\hline 22 & 20.7 & 594 & 140 & 9 \\
\hline
\end{tabular}

Se realizó un corte transversal al cordón de soldadura, el cual fue maquinado y pulido a espejo por el método tradicional para realizar perfiles de microdureza de acuerdo a la designación ASTM E-384. Se empleó una carga de $981 \mathrm{mN}(0.1 \mathrm{~kg})$ aplicada durante $15 \mathrm{~s}$ cada 200 $\mu \mathrm{m}$, hasta una distancia de $5 \mathrm{~mm}$ a partir del centro del cordón. A la misma muestra se le realizó un ataque químico por inmersión con Nital al 3\%. 6

Para obtener el comportamiento esfuerzo-deformación de la junta soldada se realizaron 3 pruebas de tensión y se registró el promedio. La Figura 3 muestra la forma de las probetas de tensión.

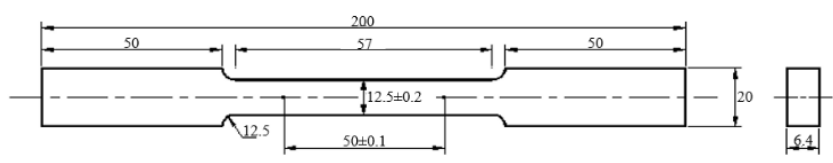

Figura 3. Geometría de las probetas de tensión de acuerdo a la norma ASTM B557M-15. Acotaciones en $\mathrm{mm} .7$ 
Tabla 1. Composición química de los materiales empleados (\% en peso).

\begin{tabular}{cccccccccccccc}
\hline Material & $\mathrm{C}$ & $\mathrm{Mn}$ & $\mathrm{Si}$ & $\mathrm{Cr}$ & $\mathrm{Ni}$ & $\mathrm{F}$ & $\mathrm{Mo}$ & $\mathrm{V}$ & $\mathrm{S}$ & $\mathrm{Cu}$ & $\mathrm{Al}$ & $\mathrm{Ti}$ & $\mathrm{Fe}$ \\
\hline $\mathrm{M}$ & 0.141 & 1.67 & 0.107 & 0.027 & 0.241 & 0.015 & 0.003 & 0.001 & 0.007 & 0.022 & 1.82 & 0.002 & 96.2 \\
$\mathrm{BC}$ & 0.003 & 0.087 & 0.006 & 0.022 & 0.008 & 0.13 & 0.002 & 0.001 & 0.006 & 0.014 & 0.004 & 0.019 & 99.8 \\
Electrodo & 0.08 & 1.45 & 0.84 & 0.025 & 0.13 & - & 0.002 & 0.002 & - & 0.12 & - & - & - \\
\hline
\end{tabular}

Se realizaron pruebas de impacto sobre los materiales base y la soldadura obtenida. Los ensayos se realizaron por triplicado y se reportan los promedios. Estos ensayos se realizaron en una torre de caida libre y los parametros utilizados se muestran en la Tabla 3. La Figura 4 muestra el arreglo experimental para este ensayo.

Tabla 3. Variables físicas de las pruebas de impacto del acero martensítico, acero BC y de la soldadura.

\begin{tabular}{ccccc}
\hline $\begin{array}{c}\text { Altura } \\
(\mathbf{m})\end{array}$ & $\begin{array}{c}\text { Masa } \\
(\mathbf{k g})\end{array}$ & $\begin{array}{c}\mathrm{E}_{\mathrm{p}} \\
(\mathbf{J})\end{array}$ & $\begin{array}{c}\mathrm{E}_{\mathrm{c}} \\
(\mathbf{J})\end{array}$ & $\begin{array}{c}\text { Velocidad } \\
\left(\mathbf{m s}^{-1}\right)\end{array}$ \\
\hline \multirow{2}{*}{1} & 14 & 137.3 & 137.34 & 4.429 \\
& & & & \\
\hline
\end{tabular}

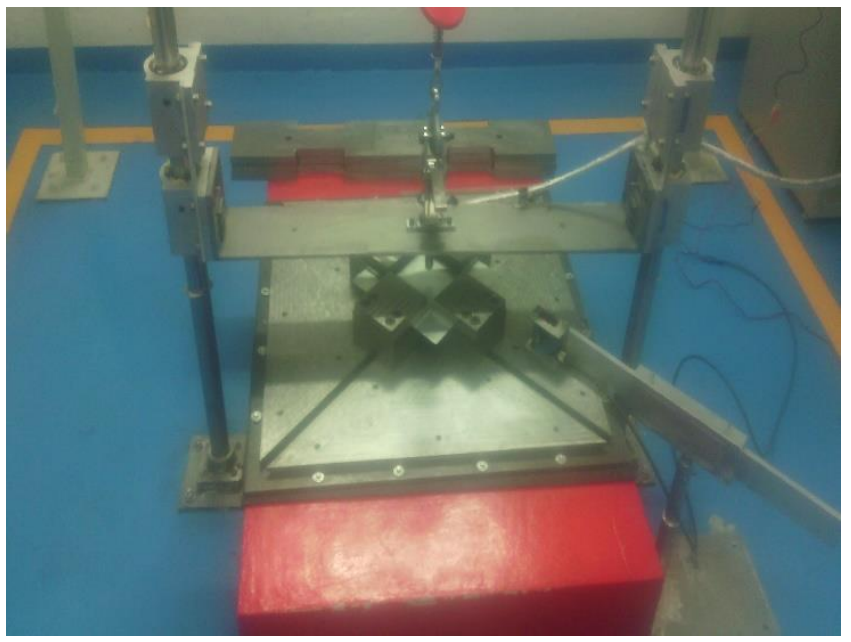

Figura 4. Arreglo experimental para el ensayo de impacto.

\section{Análisis y discusión de resultados}

La Figura 5 muestra la microestructura del acero bajo carbono (BC) donde se puede ver que consta de la fase perlita $(51.25 \%)$ y ferrita $(48.75 \%)$ que le promueve alta tenacidad. Por otro lado, la Figura 6 muestra la microestructura del acero martensítico (M) el cual presenta una matriz de martensita (98.39\%) con pequeñas cantidades de austenita retenida $(1.61 \%)$ que le brindan una alta dureza. Las características que proveen las fases mencionadas pueden verse en la Figura 7 y 8 .

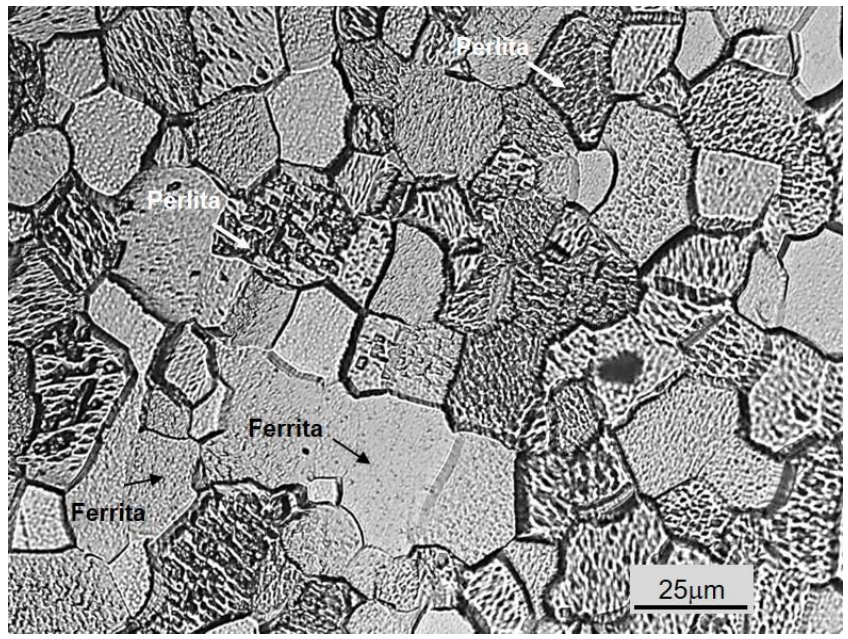

Figura 5. Microestructura del acero bajo carbono.

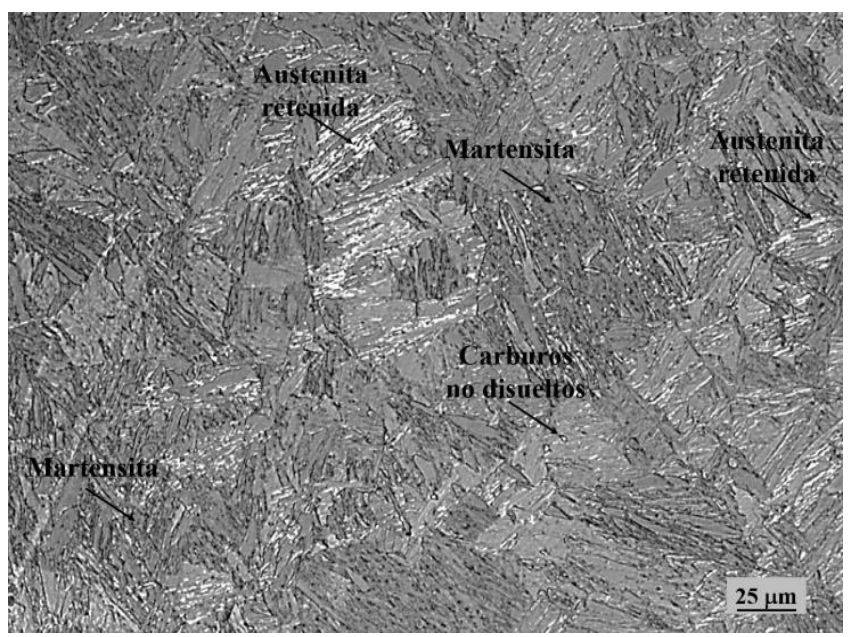

Figura 6. Microestructura del acero martensítico.

Como se mencionó, el acero bajo carbono presenta buena ductilidad, esto se puede identificar en su deformación máxima (0.6), sin embargo, presenta menor resistencia a la tensión $(280 \mathrm{MPa})$ comparado con el acero martensítico (1185 Mpa). No obstante, este último material presenta poca deformación $(0.13)$, lo que indica que su ductilidad es baja.

Los materiales caracterizados anteriormente fueron unidos por el proceso GMAW. La Figura 9 presenta la unión disímil, con un cordón de soldadura uniforme. 


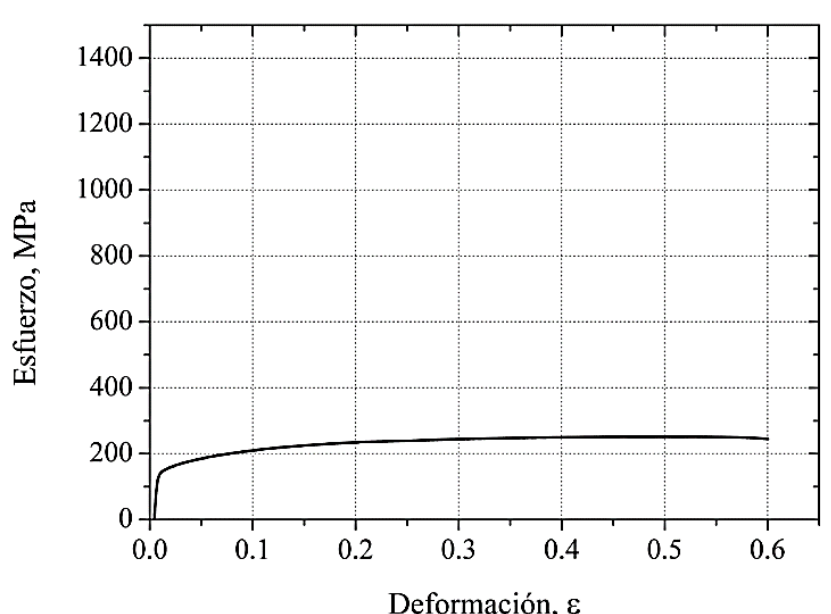

Figura 7. Resistencia a la tensión del acero bajo carbono.

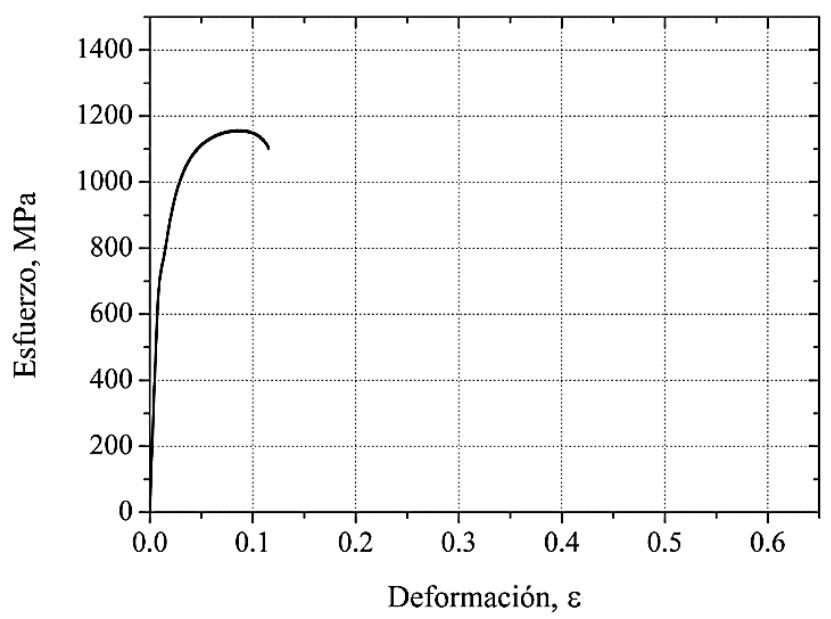

Figura 8. Resistencia a la tensión del acero martensítico.

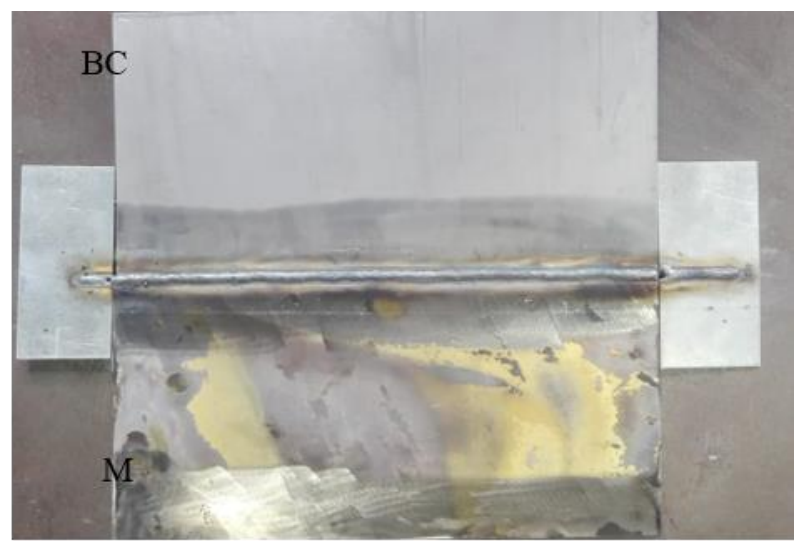

Figura 9. Unión disímil.

De la figura anterior se obtuvieron cortes transversales al cordón de soldaduras para obtener los estudios de microdureza y de tensión. La Figura 10 muestra el perfil de microdureza de la unión disímil.

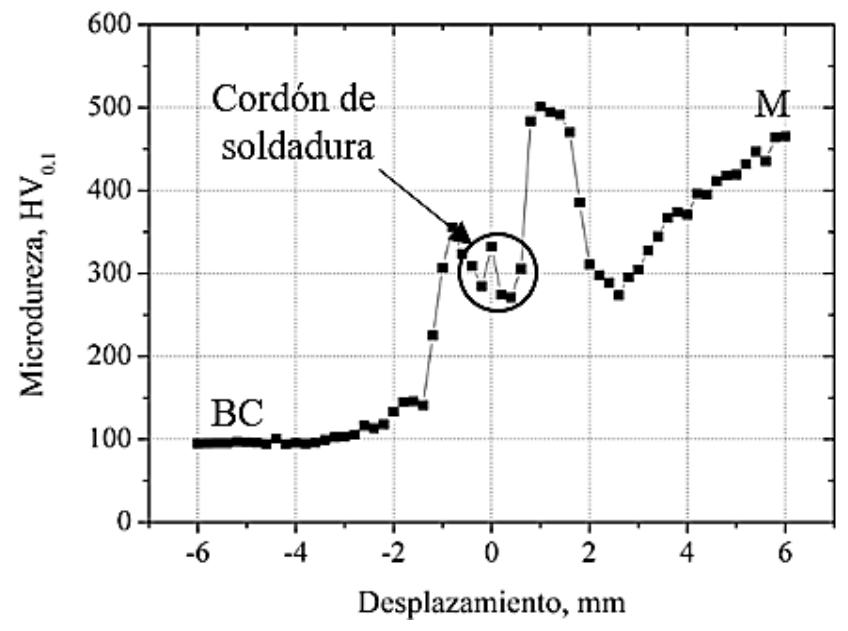

Figura 10. Perfil de microdureza de la unión disímil.

De la figura anterior se puede observar que existe un efecto sobre la microestructura junto a la zona cercana al cordón de soldadura, la cual se denomina zona afectada térmicamente.

La Figura 11 muestra los cambios microestructurales generados por el calor de aporte y que se ven reflejados en la Figura 10. En la zona $A$ se puede visualizar la microestructura ferrita-perlita del acero bajo carbono mientras que en la zona B se logra observar un crecimiento de grano y un aumento en la fase perlita que genera un aumento de dureza como se puede observar en la Figura 10 a una distancia de aproximadamente 0.8 $\mathrm{mm}$. Por otro lado, la zona D presenta el cordón de soldadura con una microestructura típica de solidificación mientras que la zona $\mathrm{C}$ y $\mathrm{E}$ presentan las interfases entre el cordón de soldadura y los materiales base donde se puede observar un crecimiento columnar. La zona $\mathrm{F}$ presenta un crecimiento de grano con una estructura netamente martensítica lo que brinda una alta dureza a 1 $\mathrm{mm}$ lo que disminuye la ductilidad en gran medida. En la zona $G$ se lleva a cabo un refinamiento de grano y empieza a precipitar la ferrita por lo que disminuye la dureza y, finalmente se tiene la zona $\mathrm{H}$ denominada zona subcrítica debido a que presenta la menor dureza y es debido a que se tiene mayor presencia de ferrita y los carburos presentan un mayor tamaño.

La Figura 12 muestra la resistencia a la tensión de la unión disímil y la Tabla 4 muestra un concentrado de los resultados del ensayo de tensión. Como se puede observar, la resistencia máxima alcanzada es la del acero de bajo carbono. Lo anterior concuerda con los resultados de la Figura 10, donde la dureza mínima alcanzada se localiza en la zona del acero al carbono. Como se puede observar en la Figura 13, la falla ocurrió en el acero bajo carbono, sin embargo, se puede ver en la Figura 12 el efecto de la unión disímil sobre la ductilidad debido a que ésta disminuye. 

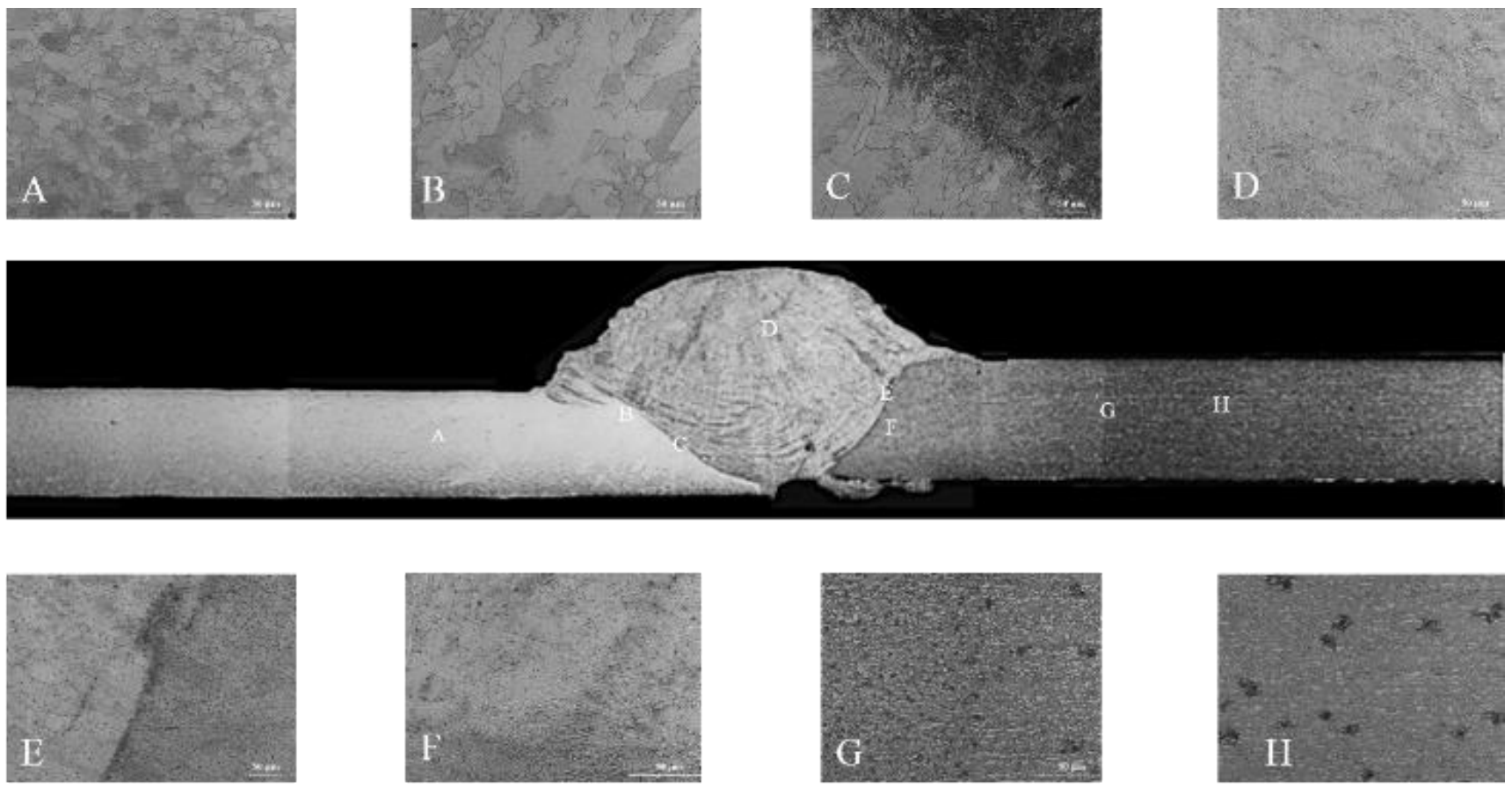

Figura 11. Zonas con distintos cambios microestructurales en la unión disímil, A) acero bajo carbono; B) crecimiento de grano ferrítico-perlítico; C) interfase cordón de soldadura-BC; D) cordón de soldadura; E) interfase cordón de soldadura$\mathrm{M} ; \mathrm{F})$ crecimiento de grano martensítico; G) refinamiento de grano $\mathrm{H}$ ) zona subcrítica.

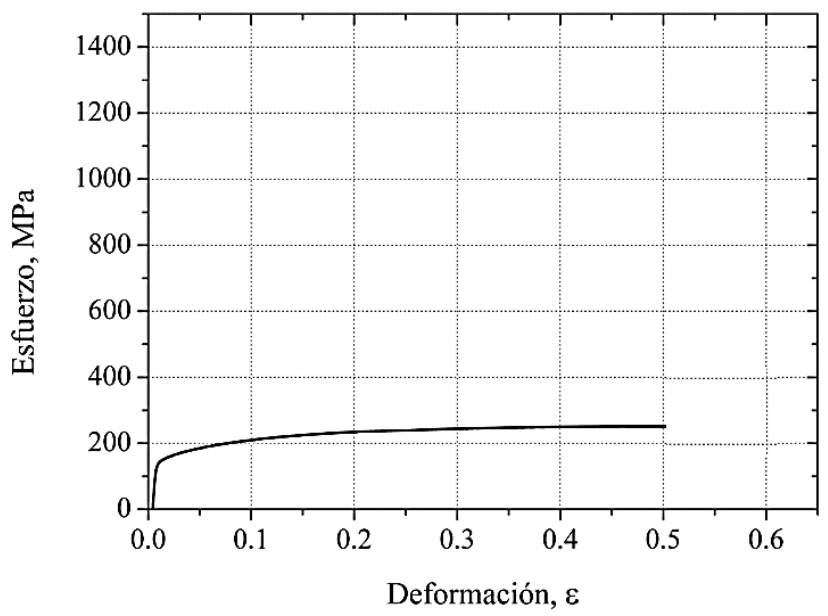

Figura 12. Resistencia a la tensión de la unión disímil.

Tabla 4. Esfuerzos y deformaciones máximas obtenidos de los ensayos de tensión de diferentes probetas.

\begin{tabular}{cccc}
\hline & $\mathbf{B C}$ & $\mathbf{M}$ & $\mathbf{B C}-\mathbf{M}$ \\
\hline$\sigma_{\text {max. }}(\mathbf{M P a})$ & 280 & 1185 & 286 \\
$\boldsymbol{\varepsilon}$ & 0.6 & 0.13 & 0.5 \\
\hline
\end{tabular}

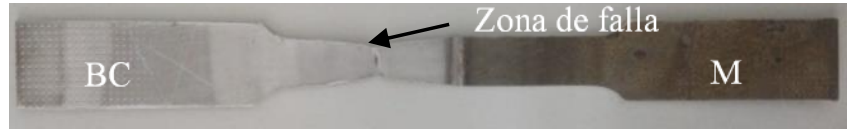

Figura 13. Probeta ensayada a tensión.

Con respecto a los ensayos de impacto las Figuras 14, 15 y 16 muestran la evolución de la fuerza que opone el material durante el impacto en función del tiempo, del acero bajo carbono, martensítico y de la unión disímil respectivamente. Como se logra apreciar el material que menos fuerza opuso fue el acero BC con aproximadamente $35 \mathrm{kN}$, mientras que el acero martensítico mostró la mayor fuerza $(48 \mathrm{kN})$. Como se logra apreciar en la unión disímil, el comportamiento de la unión soldada es superior al acero bajo carbono, esto se atribuye a la diferente composición de fases que presenta el cordón de soldadura junto con las ZAT de los distintos materiales respecto a los materiales fases como se puede apreciar en la Figura 11.

Durante el ensayo de impacto un sensor laser fue colocado en la parte inferior de las láminas, éste fue alineado con el percutor (punzón de impacto) para medir el desplazamiento del material en el punto de impacto durante el ensayo. Las Figuras 17, 18 y 19 muestran los resultados. El desplazamiento máximo generado fue de 
4.8, 5.23 y $7.1 \mathrm{~mm}$ para el acero bajo carbono, acero martensítico y la soldadura respectivamente.

Para determinar la energía absorbida durante el impacto se utilizó la Ecuación 1.

$$
E=V_{0} \int_{0}^{t} F(t) d t
$$

Donde la integral representa el área bajo la curva del comportamiento fuerza-tiempo y $V o$ es la velocidad de deformación que se obtiene con el desplazamiento máximo y el tiempo total del evento.

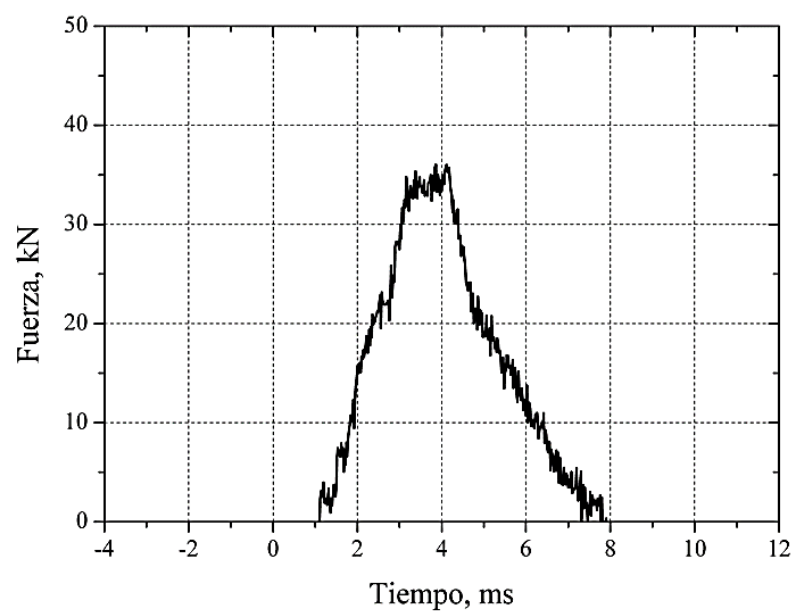

Figura 14. Comportamiento fuerza-tiempo del acero bajo carbono durante el tiempo de ensayo.

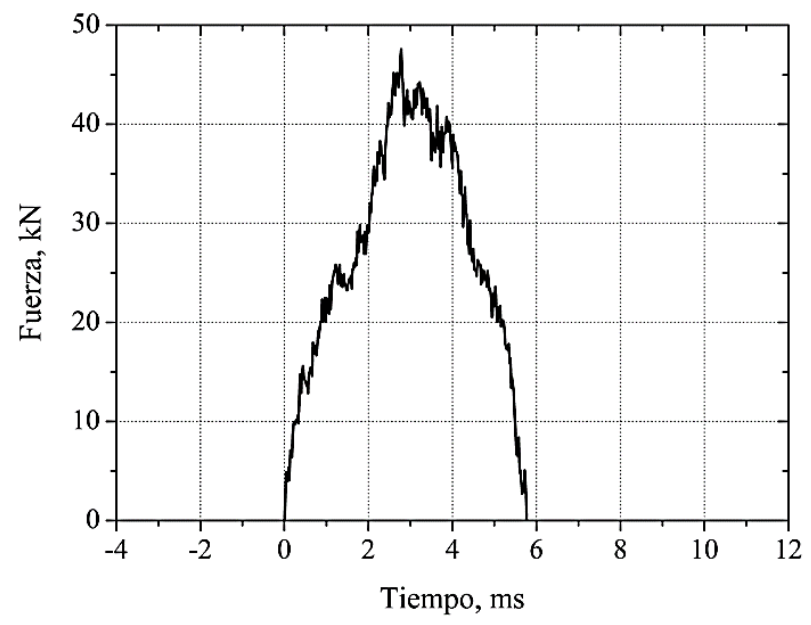

Figura 15. Comportamiento fuerza-tiempo del acero martensítico durante el tiempo de ensayo.

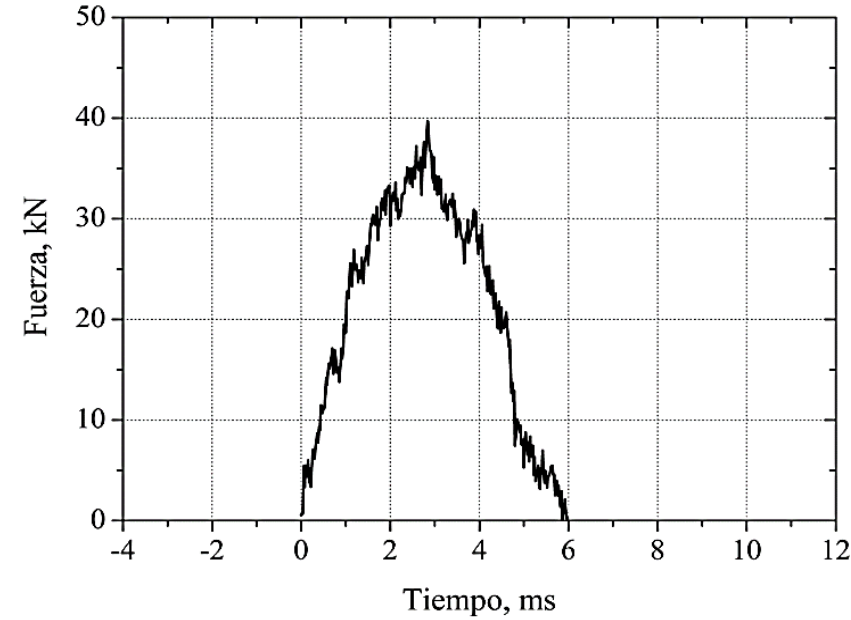

Figura 16. Comportamiento fuerza-tiempo de la soldadura durante el tiempo de ensayo.

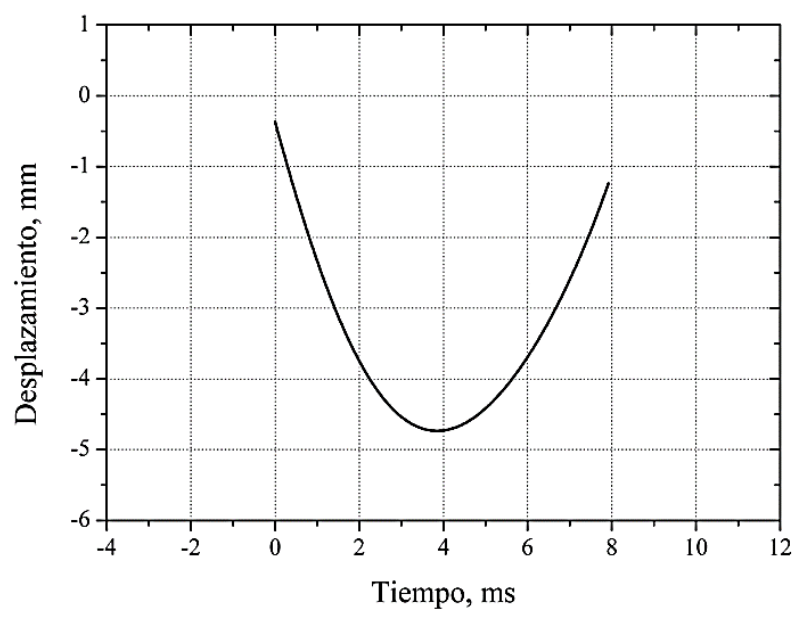

Figura 17. Curva desplazamiento-tiempo de la prueba de impacto en un acero bajo carbono.

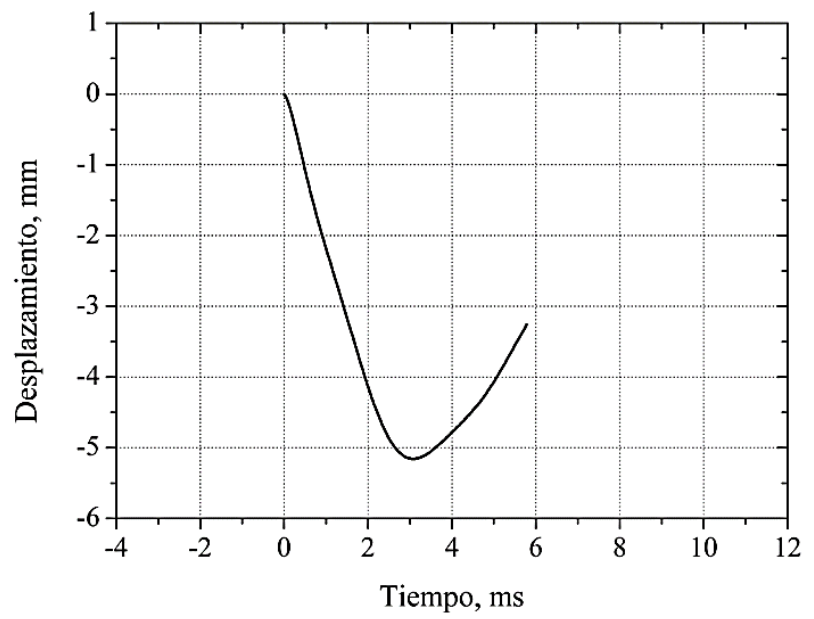

Figura 18. Curva desplazamiento-tiempo de la prueba de impacto en un acero martensítico. 


\section{Referencias}

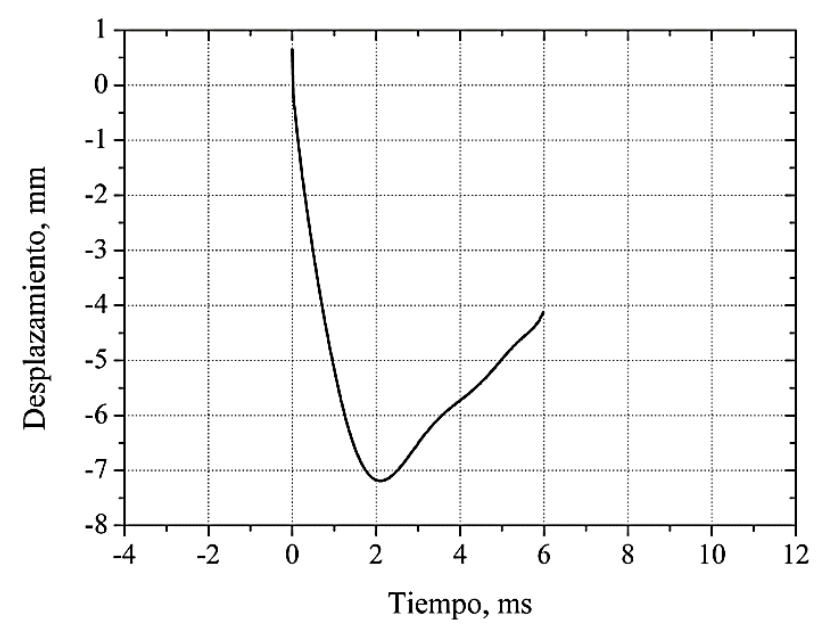

Figura 19. Curva desplazamiento-tiempo de la prueba de impacto en la unión disímil BC-M.

Aplicando la Ecuación 1 en cada condición se pudo cuantificar la energía absorbida por el acero bajo carbono $(66.25 \mathrm{~J})$, acero martensítico $(127.72 \mathrm{~J})$ y la soldadura (136.27 J).

La condición de soldadura mostró mejor comportamiento en lo que a absorción de energía se refiere. Sin embargo, se recomienda realizar estudios adicionales de impacto a distintos niveles de energía ya sea variando la masa del percutor o la altura para cuantificar el comportamiento al impacto hasta llevar al material a la fractura.

\section{Conclusiones}

EI WPS planteado cumple con las condiciones necesarias para la unión disímil BC-M. Logró obtenerse un cordón uniforme de penetración completa. El calor de aporte generó cambios microestructurales sobre la zona aledaña al cordón de soldadura (zona afectada térmicamente), sin embargo, no causaron la disminución de la resistencia a la tensión por debajo del material de menor resistencia (BC). La unión disímil genera una disminución sobre la deformación con respecto al acero al carbono. La soldadura absorbió la mayor cantidad de energía durante el impacto, no obstante, es indispensable realizar estudios de impacto adicionales hasta llevar a la fractura a la muestra.

\section{Agradecimientos}

Los autores agradecen al Programa para el Desarrollo Profesional Docente (PRODEP) por el apoyo brindado para realizar esta investigación
[1] Hilditch TB, Souza TD, Hodgson PD. Properties and automotive applications of advanced high-strength steels (AHSS). Materials characterization 2016; 112: 229-237.

[2] Gould JE, Khurana SP, Li T. Predictions of microstructures when welding automotive advanced high-strength steels. Welding Journal 2006; 111-116.

[3] Oliver ST, Jones B, Fourlaris. Dual phase versus TRIP strip steels: microstructural changes as a consequence of quasi-static and dynamic tensile testing. Materials characterization 2007; 58: 390-400.

[4] AISI. Ultralight steel auto body -advanced vehicle concepts report / AISI 2002.

[5] AWS D1. 1M: 2010. Código de soldadura estructural- Acero. 2010.

[6] ASTM E384, Standard Test Method for Microidentation Hardnees of Materials; 2004; 384-399.

[7] ASTM E8M, Standard Test Methods for Tension Testing of Metallic Materials 2004; 1-27. 